\title{
Algunas Reflexiones en Clave de Gestión Tras las Primeras \\ Defensas de Trabajos Fin de Grado
}

\author{
Ángel Elias Ortega*
}

\section{RESUMEN:}

En este primer año de los TFG ha habido unas 25 defensas públicas. Esta comunicación versa sobre la experiencia y posterior reflexión, especialmente en lo ligado a los mecanismos de gestión.

Las pautas generales venían marcadas por la propia titulación, ya que la normativa marco de la UPV/EHU no ha sido aprobada hasta el pasado 3 de marzo de 2011. De cara a visibilizar mejor todo el proceso de coordinación seguido se indican en la comunicación los principales hitos, es decir, las sucesivas fases, reuniones y documentos elaborados.

Aunque la valoración del proceso se considera en conjunto muy positiva, son sin embargo muchos los aspectos que pueden mejorarse. Por tal motivo se plantean diversas reflexiones que se concretan a modo de sugerencias.

\section{Palabras Clave:}

Trabajo Fin de Grado, Gestión, Reconocimiento, Sugerencias

\section{ABSTRACT:}

During this first year of the Degree Theses there have been around 25 public defenses. This paper discusses this experience and its later reflection particularly regarding its management mechanisms.

* Departamento Derecho de la Empresa. Universidad del País Vasco • angel.elias@ehu.es 
The general guidelines had been determined by the correspondent university degree, since the UPV/EHU current regulation has not been approved until the $3^{\text {rd }}$ March 2011. In order to discern the whole coordination process that has been followed, in this paper there have been outlined the main milestones, i.e. the consecutive stages, meetings and drawn up documents.

Although the assessment of this process has been considered highly positive as a whole, there are many aspects that can still be improved. This is the reason why we present some thoughts as suggestions for improvement.

\section{KEYWORDS:}

Degree Thesis, Management, Recognition, Suggestions

\section{1.- INTRODUCCIÓN}

El pasado curso académico 2010/11 ha sido el primer año de esta titulación en la UPV/EHU, en el que además se ha podido matricular y defender los Trabajos Fin de Grado, en lo sucesivo TFG, en dos convocatorias. La primera se ha celebrado en julio de 2011 con 6 defensas públicas, y la segunda tendrá lugar la semana del 19 de septiembre con aproximadamente otras 20 defensas públicas.

Las pautas generales venían marcadas por la propia titulación. Concretamente en el Plan de Estudios el TFG aparece ubicado en el quinto y último módulo como una materia obligatoria, con una carga lectiva de 6 créditos, equivalente a 150 horas de trabajo. El TFG se plasma en una investigación académico-profesional que exige la aplicación por parte del alumno/a de sus conocimientos, dotes de creatividad y originalidad, e implica una prueba de madurez antes de iniciar el ejercicio profesional. Se espera que las/os estudiantes sean capaces de conseguir las siguientes competencias:

- Aplicación de las competencias adquiridas durante el grado a la realidad de las relaciones laborales y de los recursos humanos, reflejando los principios deontológicos de la profesión relacionada con el objeto del trabajo.

- Síntesis, integración e interrelación de las diversas competencias plasmándolas en el TFG.

- Elaboración de un trabajo con calidad y apoyo teórico sólido, ordenado, que aborde todos los aspectos del tema elegido y plasme una sensibilidad informada ante realidades de desigualdad y exclusión.

- Defensa sólida del trabajo presentado exponiéndolo y respondiendo a las posibles preguntas con claridad y coherencia. 
La normativa marco de la UPV/EHU no ha sido aprobada hasta el pasado 3 de marzo de $2011^{1}$. Por ello, la dirección del centro es la que ha impulsado el proceso de coordinación de los TFG, habiendo establecido un plan de trabajo con el profesorado implicado en el mismo, sobresaliendo la participación de un grupo de 8 profesoras/ es, que han formado un equipo docente. Ha sido precisamente este equipo docente el que ha ido proponiendo, por consenso, la regulación del proceso de elaboración y defensa de los TFG, que posteriormente ha sido analizado por la Comisión de Ordenación Académica y presentado por la Dirección para su aprobación por la Junta de Centro el 14 de abril de 2011 (en anexo 1).

\section{2.- PROCESO SEGUIDO}

De cara a visibilizar mejor todo el recorrido de este proceso, procedo a indicar a continuación los principales hitos, es decir, las sucesivas fases, con sus reuniones y documentos elaborados. Así:

1. La dirección de los TFG es una obligación compartida por todos los Departamentos, estando obligados a asumir, si fuera necesario, el número de TFG proporcional al $\mathrm{n}^{\circ}$ de créditos que cada Departamento imparte en la titulación. Por ello el 20/9/2010 se pide a las/os coordinadores de los 13 diferentes Departamentos que tienen docencia en este grado la remisión, para antes del 8 de octubre de 2010 , de la propuesta de temas que proponen, indicando el nombre del director/a de cada tema.

2. El $27 / 10 / 10$ se remite a las 40 personas matriculadas en TFG la anterior relación de temas, pidiéndoles que antes del 28/11/10 indiquen por orden sus primeras cinco preferencias o, en su caso, aporten otro tema que cuente con el aval de algún profesor/a del centro y que asuma dirigirlo.

3. El 1/12/10, aprovechando una reunión con la vicerrectora de Innovación Educativa, se convoca a todo el profesorado para comentar lo relativo al proceso que se estaba siguiendo en los TFG.

4. El 9/12/10 se comunica a cada alumna/o matriculado el tema y director de su TFG. Para ello se favorece su preferencia, de forma que de las 40 personas matriculadas en 34 casos coincide con su primera elección de temas $\mathrm{y}$, en las otras 6 personas con su segunda preferencia. El lado negativo lo constituye que las preferencias temáticas del alumnado no alcanzaron a la totalidad de los Departamentos, siendo destacable las facilidades dadas por

1. Puede verse dicha normativa en la web http://www.lan-harremanak.ehu.es/p231-content/es/contenidos/informacion/rrll_grado/es_grado/adjuntos/anexo_4.pdf 
el profesorado, que en muchos casos estuvo dispuesto a dirigir el TFG de más de una persona.

5. El 20/12/10 se celebra una sesión formativa de 3 horas impartida por la profesora Itziar Rekalde, experta en temas de innovación educativa, que versa sobre cómo evaluar competencias en la elaboración del TFG, así como pautas para una guía docente del TFG. Asisten 12 profesoras/es del centro.

6. Gracias en buena medida a la formación recibida se elabora un documento sobre la estructura, cronograma y evaluación del TFG, que se remite a todo el profesorado y alumnado implicado el 25/1/11.

7. El 14/3/11 la vicerrectora de Ordenación Académica de la UPV/EHU remite al centro la normativa de TFG que ha sido aprobada por el Consejo de Gobierno de la UPV/EHU el anterior 3/3/11. Tras ello se convoca a todo el profesorado que está dirigiendo TFG a una reunión que se celebra el 29/3/11 y en la que se analiza los cambios que la nueva normativa incorpora y se consensuan los criterios para su desarrollo en nuestra titulación.

8. El 4/4/11 se envía a todo el profesorado que está dirigiendo TFG la propuesta de desarrollo normativo del TFG en nuestra titulación. Dicha propuesta se trabaja en la reunión de la Comisión de Ordenación Académica del centro celebrada el 11/4/11, donde se incorporan diversas mejoras, algunas de ellas fruto de la aportación de la representante del alumnado, que es aprobada por Junta de Centro celebrada el 14/4/11.

9. El 19/4/11 se celebra una reunión con el alumnado matriculado en TFG y se comenta la nueva situación originada por la normativa de la UPV/EHU y el desarrollo de la misma aprobado por la Junta del Centro. La principal consecuencia es que de las 40 personas matriculadas varias de ellas no cumplen el requisito de estar matriculadas en todos los demás créditos del grado, ante lo que se plantea la posibilidad de subsanar la situación o, en su caso, renunciar a la matricula del TFG y obtener la devolución de las tasas, cosa que acontece con 13 personas, de manera que el número de matriculadas se queda finalmente en 27 personas.

10. El 16/5/11 se solicita a las/os coordinadores de Departamentos que antes del 24/5/11 indiquen las/os miembros de cada Tribunal de Evaluación.

11. El 31/5/11 se informa al alumnado matriculado, así como al profesorado afectado, de la composición de cada Tribunal de Evaluación, así como de las fechas en que deberán solicitar su defensa en las convocatorias de julio y septiembre de 2011.

12. El $15 / 6 / 11$ se envía una convocatoria de reunión para el siguiente $24 / 6 / 11$ a todo el profesorado afectado en los distintos Tribunales de Evaluación de 
los TFG, a fin de compartir los diferentes aspectos que rodean la celebración de las defensas públicas de los TFG.

13. Los días 5 y 6 de julio de 2011 se desarrollan las 6 defensas públicas, que obtienen las calificaciones siguientes: 10, 10, 9, 9, 7,5 y 7. Dado su pequeño número, será importante esperar a conocer los resultados de las defensas de la $3^{\text {a }}$ semana de septiembre para tener una visión más global de los resultados obtenidos.

14. En todo este proceso, además de la comunicación directa a las personas afectadas, tanto alumnado como profesorado, se ha procedido a dar una continuada información en el apartado NOTICIAS de la web, así como publicado cada uno de los documentos en el apartado de la web referente a los TFG (http:// www.lan-harremanak.ehu.es/p231-content/es/contenidos/informacion/rrll_grado/es_grado/grado.html ). Junto a ello se han colocado diversos anuncios en los tablones de las clases afectadas y en el hall del centro.

\section{3.- ALGUNAS REFLEXIONES}

A falta todavía de una valoración más completa, que se realizará este mismo mes de septiembre tras la finalización de las correspondientes defensas públicas, planteo seguidamente diversas reflexiones, que seguramente podrán permitir la introducción de algunos cambios cara a una mejora del proceso. Así:

1. La creación de un registro para solicitar el inicio del proceso del TFG. De esta forma antes o a la vez de la matriculación del TFG se procederá a admitir el registro de la solicitud de iniciar el proceso de elaboración del TFG. Entre otras ventajas dará flexibilidad a la hora del cumplimiento estricto de los requisitos para la matriculación, ya que alumnado que está en proceso de realización de prácticas u otras actividades que permiten la convalidación de créditos, no cumple el requisito de tener matriculadas todas las asignaturas requeridas para finalizar el plan de estudios2.

2. Que la relación de temas y profesorado que vaya a dirigirlos esté publicado en el mes de julio de cada año, antes del proceso de matriculación de los TFG.

3. Incorporar entre la documentación nuevas plantillas como anexos, concretamente una referente a la evaluación de la defensa y otra a la autorización para su publicación (anexos 3 y 4, respectivamente).

2. Requisito exigido por el art. 3.1 de la normativa de la UPV/EHU, que regula el Trabajo Fin de Grado (anexo 1), y el párrafo $2^{\circ}$ del art. 2 de la Normativa de Desarrollo, aprobada por la Junta de Centro de la E.U. de Relaciones Laborales (anexo 2). 
4. Indicar que normalmente, salvo que el Tribunal no lo considerase conveniente, a la terminación de la defensa y de manera similar a lo que acontece con las tesis, el Tribunal ordenará que se desaloje la sala para poder deliberar, tras lo cual llamará nuevamente a vista pública para proceder a comunicar en forma solemne la calificación otorgada al TFG.

5. Plantear que igualmente se lleve la presentación en formato pdf, ya que las diferentes versiones del powerpoint son en ocasiones incompatibles con el programa del ordenador, y que se entreguen en secretaría 5 ejemplares del TFG en papel.

6. Que sea posible causa de abstención por parte del Director/a del TFG a fin de no formar parte como Secretaria/o del Tribunal y ser sustituida/o por la/ el suplente los supuestos en que la calificación otorgada a la elaboración del TFG no llegue al aprobado.

7. La instauración de un premio por parte de los Colegios de Graduados Sociales de Euskadi al TFG mejor valorado de entre los que tengan alguna relación con el perfil profesional del/la graduada social3.

8. Insistir ante las correspondientes instancias de la UPV/EHU que la dirección del TFG debe ser computada en mucha mayor medida que la reconocida actualmente4, proponiendo que al menos se le aplique el mismo ratio que a los másteres, es decir, el doble. Ello es debido a que en el grado es necesario un mayor apoyo y seguimiento al alumnado, lo que deriva en una importante dedicación de tiempo.

Leioa a 8 de septiembre de 2011.

3. Ya hay un acuerdo con los tres Colegios de Graduados Sociales de la C.A. del País Vasco para que se instaure este mismo año.

4. De acuerdo con la normativa vigente (Consejo de Gobierno de la UPV/EHU de 19 de noviembre de 2009) en esta universidad, la dirección de un TFG de 6 créditos computa como 0,25 créditos de encargo docente por trabajo dirigido. Éstos se cargan al profesorado que dirige TFG con un diferimiento de dos cursos académicos. 


\section{ANEXO 1}

DESARROLLO DE LA NORMATIVA SOBRE LA ELABORACIÓN Y DEFENSA DEL TRABAJO FIN DE GRADO

\section{1.- INTRODUCCIÓN}

La presente normativa es un desarrollo de la aprobada por el Consejo de Gobierno de la UPV/EHU el 10 de marzo de 2011, en relación siempre con lo establecido en la titulación de este Grado de Relaciones Laborales y Recursos Humanos. Su aprobación por la Junta de Centro ha venido precedida de la consulta con el profesorado participante el curso 2010/11 en la dirección de los Trabajos Fin de Grado, en lo sucesivo TFG, así como del debate en la Comisión de Ordenación Académica del Centro.

En el Plan de Estudios el TFG aparece ubicado en el quinto y último módulo como una materia obligatoria, con una carga lectiva de 6 créditos, equivalente a 150 horas de trabajo. El TFG se plasma en una investigación académico-profesional que exige la aplicación por parte del alumno/a de sus conocimientos, dotes de creatividad y originalidad, e implica una prueba de madurez antes de iniciar el ejercicio profesional.

Se espera que los/as estudiantes sean capaces de conseguir las siguientes competencias:

- Aplicación de las competencias adquiridas durante el grado a la realidad de las relaciones laborales y de los recursos humanos, reflejando los principios deontológicos de la profesión relacionada con el objeto del trabajo.

- Síntesis, integración e interrelación de las diversas competencias plasmándolas en el TFG.

- Elaboración de un trabajo con calidad y apoyo teórico sólido, ordenado, que aborde todos los aspectos del tema elegido y plasme una sensibilidad informada ante realidades de desigualdad y exclusión.

- Defensa sólida del trabajo presentado exponiéndolo y respondiendo a las posibles preguntas con claridad y coherencia.

\section{2.- INSCRIPCIÓN Y CONVOCATORIAS}

La inscripción se realizará dentro del plazo general de matrícula que establezca la UPV/EHU. No obstante, dado que el TFG se ubica en el segundo cuatrimestre, se habilitará un plazo de matriculación en torno al inicio de dicho segundo cuatrimestre.

Para matricularse en el TFG será necesario tener matriculadas todas las asignaturas requeridas para finalizar el plan de estudios, así como no tener más de 12 
créditos obligatorios pendientes de aprobar de entre los módulos primero, segundo y tercero del grado5.

La matrícula dará derecho a dos convocatorias oficiales en cada curso académico, considerándose que el mismo se inicia en octubre y termina en septiembre. El alumnado que no haya sido evaluado o no haya defendido su trabajo en las convocatorias establecidas para el curso deberá volver a matricularse en el siguiente curso académico.

Finalizado el plazo de matrícula la Secretaría enviará al profesor/a coordinadora del módulo del TFG el listado de alumnado matriculado en la asignatura.

La defensa de los TFG será de forma distribuida a lo largo del curso académico, concretamente alrededor de las dos últimas semanas de los meses de noviembre, marzo, junio y septiembre, en fechas que se comunicarán debidamente.

\section{3.- Asignación de trabajos Realizables y diReCción de los mismos}

La dirección del centro solicitará a los departamentos la oferta de TFG, cuyo representante en el centro lo comunicará de acuerdo al modelo correspondiente (Anexo 1). Esta oferta, que incluirá el profesor/a que lo vaya a dirigir, se hará pública antes del inicio del curso, especificándose la lengua en que deba elaborarse y defenderse. A continuación hay un plazo hasta la finalización del mes de septiembre6 para que el alumnado matriculado envíe sus preferencias ordenadas de 1 a 5 por correo electrónico a la secretaría de la dirección del centro. En caso de que un mismo tema fuera solicitado por varias personas el mismo será asignado a quien tenga una mayor puntuación media en su expediente académico en esta titulación.

En este mismo plazo el alumnado también podrá presentar al profesor/a coordinadora del módulo del TFG su propuesta propia de TFG, que deberá incluir la firma de un/a profesora del centro que evalúe positivamente la propuesta y acepte ser el director/a del trabajo correspondiente, así como del Departamento al que pertenezca (Anexo 2).

En cualquier caso todo TFG tiene asociado un director/a, quien deberá enviar al profesor/a coordinadora del módulo del TFG el correspondiente plan de dirección de dicho trabajo. Podrá ser director/a de un TFG cualquiera de los profesores/as del centro. Si no hubiera profesorado voluntario suficiente en los departamentos, siem-

5. En los cursos 2010/11 y 2011/12 y en relación con el alumnado que inició la titulación como Diplomatura Universitaria en Relaciones Laborales será suficiente con tener acreditados 168 créditos.

6. Para quienes se hubieran matriculado del TFG en el segundo período, en torno al inicio del segundo cuatrimestre, este plazo será de quince días naturales a contar desde que hubiera terminado el citado período de matriculación. 
pre en proporción a la presencia de créditos que tiene cada departamento en esta titulación, designarán de entre su profesorado adscrito a este centro a quienes vayan a desarrollar esta labor7 en dicho curso.

Lo antes posible y, en cualquier caso, con anterioridad a la finalización del primer cuatrimestres se comunicará al alumnado matriculado el tema del TFG, así como el profesor/a que le dirigirá.

El profesor/a será responsable de exponer a cada estudiante las características del trabajo, de orientarlo en su desarrollo y de velar por el cumplimiento de los objetivos fijados, así como de realizar el seguimiento y elaborar un informe escrito previo a la defensa, del que se dará traslado a la estudiante o al estudiante.

\section{4.- Métodos y pautas de elaboración del TFG}

El TFG tendrá una extensión aproximada de entre 55.000 y 110.000 caracteres sin espacios.

Deberá tener calidad y apoyo teórico y versará sobre algún tema relacionado, entre otros, con aspectos históricos-sociales, de dirección y gestión de los recursos humanos, económicos o jurídicos del trabajo y las relaciones laborales.

Los aspectos formales deberán ser cuidados, de manera que el contenido esté correcta y lógicamente organizado, abordando todos los aspectos que conforman el tema elegido, siendo la redacción correcta desde el punto de vista gramatical y semántico, de manera que los contenidos aparezcan claramente expresados y la vinculación existente entre las ideas quede bien reflejada.

Además deberán incorporarse las referencias bibliográficas, de forma que las ideas tomadas de algunos autores vayan acompañadas de su correspondiente referencia y todas ellas aparezcan incluidas en el apartado de "Referencias Bibliográficas". Igualmente se realizará un resumen en inglés de al menos cien palabras del TFG.

La presentación seguirá los criterios siguientes:

1. Se escribirá en hojas DIN A4.

2. La portada deberá contener la siguiente leyenda:

7. De acuerdo con la normativa vigente (Consejo de Gobierno de 19 de noviembre de 2009) en nuestra universidad, la dirección de un TFG de 6 créditos computa como 0,25 créditos de encargo docente por trabajo dirigido. Éstos se cargan al profesorado que dirige TFG con un diferimiento de dos cursos académicos.

8. Para quienes se hubieran matriculado en torno al inicio del segundo cuatrimestre, el tema del TFG y el nombre del profesor/a que le dirigirá les serán comunicados en el plazo de quince días naturales desde que terminara el plazo que el alumnado tiene para presentar su elección. 
- Trabajo Fin de Grado

- Escuela Universitaria de Relaciones Laborales de la UPV/EHU

- Título del Trabajo

- Nombre y apellidos del alumno/a

- Nombre y apellidos del director/a del Trabajo

Cabe agrupar la diversidad de TFG en estos tres tipos:

- Trabajos de investigación empírica: en la medida de lo posible estos trabajos seguirán el modelo del método científico que proporciona una forma de actuar mediante operaciones ordenadas orientadas a la investigación, a través de una serie de fases interdependientes entre sí.

La estructura de este tipo de trabajo será la siguiente:

1. Definición del problema o tema de estudio.

2. Definición de los objetivos a alcanzar (generales y específicos).

3. Formulación de hipótesis.

4. Determinación de la metodología de recogida de datos (instrumento y fuentes).

5. Selección de la muestra objeto de estudio.

6. Recogida de datos.

7. Análisis e interpretación de los datos.

8. Resultados.

9. Interpretación de resultados y aplicación: implicaciones para la intervención en el contexto de las relaciones laborales.

10. Conclusiones

- Trabajos de investigación teórica, de carácter descriptivo y de revisión e investigación bibliográfica de temas o problemas de actualidad y de interés para los graduandos/as. En este tipo de trabajos el acento se pondrá en la revisión de la bibliografía sobre un objeto de estudio adecuado y pertinente en el contexto de las relaciones laborales en cualquiera de sus vertientes jurídica, económica, sociológica, psicosocial, organizacional e histórica, así como en la capacidad de relacionar de manera lógica, creativa y estructurada los conceptos, datos y modelos relacionados con el objeto de estudio. 
La estructura de estos trabajos, aunque no sean trabajos de investigación empírica, habrá de seguir una lógica similar a la del proceso hipotético-deductivo de investigación científica, pero obviando los aspectos relativos a la obtención y análisis de datos empíricos. El resultado de estos trabajos debería ser un informe riguroso del estado del arte y/o de integración de conceptos y modelos relativos al objeto de estudio elegido y que podría dar lugar o justificar, como tal, un trabajo de investigación empírica posterior.

Por tanto, la estructura de este tipo de trabajo será la siguiente:

1. Definición del problema o tema de estudio.

2. Definición de los objetivos a alcanzar (generales y específicos).

3. Descripción de los procedimientos de búsqueda bibliográfica

4. Descripción de las fuentes bibliográficas tanto electrónicas como impresas.

5. Análisis de los datos bibliográficos: habrá de ser el apartado más importante del trabajo y en él se expondrán de forma estructurada tanto los conceptos e ideas más relevantes del objeto de estudio como su análisis, integración y/o interpretación. Se deberá estructurar en los subapartados que sean necesarios para una exposición coherente y ordenada de conceptos que nos permita alcanzar los objetivos propuestos en el apartado 2.

6. Propuestas de intervención o de otros estudios empíricos: el alumno/a, sobre la base del análisis de la bibliografía realizado, deberá proponer algún tipo de análisis o intervención empírica que se derive de la interpretación del contenido bibliográfico analizado y desarrollado en el apartado 5.

7. Conclusiones

- Trabajos prácticos de carácter profesional: Este tipo de trabajos se centrará en la realización de un informe que recoja la descripción de un caso práctico de tipo profesional perteneciente a cualquier dominio de las relaciones laborales. Deberá describir de forma específica y detallada los siguientes aspectos del trabajo práctico:

1. Tema, objeto y nombre de la práctica.

2. Campo de las relaciones laborales en el que se inscribe la práctica y análisis bibliográfico y de documentación.

3. Descripción de la organización, empresa o institución en el contexto donde se realiza la práctica

4. Descripción y análisis detallado de la práctica: incluirá todos los subapartados necesarios para describir de forma suficiente y satisfactoria la práctica objeto del trabajo fin de grado. 
5. Conclusiones.

6. Implicaciones para la intervención en las relaciones laborales

5.- ReLACIÓN CON EL DIRECTOR/A Y EVALUACIÓN DEL PROCESO DE ELABORACIÓN.

Durante el proceso de realización del TFG el alumno/a deberá de mantener varias reuniones con el director/a del trabajo, pudiendo servir de referencia las siguientes fases:

a) Diseño del proyecto de TFG: Antes de comenzar el desarrollo del trabajo como tal cada alumno/a deberá presentar a su director/a un proyecto en el que, en un folio y de forma sintética, definirá el trabajo a desarrollar indicando en los apartados correspondientes:

- Nombre del alumno/a

- Nombre del director/a

- Título del TFG

- Justificación y objetivos

- Breve descripción de la metodología (trabajo de investigación empírica o bibliográfica) o proceso práctico (trabajo de tipo práctico)

- Bibliografía o fuentes documentales

El director/a explicará al alumno/a las diferentes partes de este proyecto y le ayudará a:

- Clarificar el marco del TFG

- Definir la estructura del TFG

- Facilitar la información de partida

- Establecer las pautas formales a seguir en el TFG

- Explicar los criterios de evaluación

- Fijar el calendario de las siguientes reuniones de tutorización

b) Presentación del proyecto de TFG: tendrá lugar entre una y dos semanas después de la primera reunión con el director/a, quien dará recomendaciones al alumno/a para mejorar el proyecto del TFG en aquellos aspectos en que lo considere necesario. Una vez que se da el visto bueno al proyecto comienza la elaboración del TFG como tal, tomando como referente constante el proyecto realizado. El desarrollo del trabajo podría desviarse del proyecto inicial si así lo requiriese su correcta realización, aunque el proyecto habrá de ser siempre el referente del alumno/a para realizar su trabajo. 
c) Seguimiento del desarrollo del TFG (1): esta primera reunión sobre el desarrollo del TFG tendrá lugar unas dos semanas después del visto bueno del proyecto y en ella el director/a:

- Verificará que el contenido, la estructura, la profundidad, la extensión y el formato del TFG es el adecuado.

- Identificará los problemas que existen y dará recomendaciones al alumno/a para solventarlos.

- Asistirá al alumno/a para resolver sus dudas.

- Se pondrá de acuerdo con el alumno/a sobre las tareas a realizar en la prosecución del desarrollo del trabajo y propondrá una fecha para la próxima reunión de seguimiento.

d) Seguimiento del desarrollo del TFG (2): esta segunda reunión de seguimiento tendrá lugar unas tres semanas después de la anterior y en ella el director/a deberá:

- realizar las mismas tareas que en la anterior

- dar al alumno/a las recomendaciones adecuadas para la mejora del trabajo de cara a su finalización

- $\quad$ poner fecha para la próxima y última reunión de seguimiento.

e) Revisión final del TFG: Tendrá lugar unas tres semanas después de la última sesión y en ella el director/a realizará:

- la revisión final del TFG, comprobando que se han llevado a cabo correctamente todas las indicaciones pactadas con el alumno/a en el desarrollo de su trabajo.

- las recomendaciones de mejora que sean necesarias para una correcta finalización de la memoria del TFG y de su presentación.

- una valoración del proceso de elaboración del TFG (40\% del total), que será realizado por escrito de acuerdo con la plantilla que aparece en el anexo 3, en función de tres criterios: elección del tema, planificación y desarroll. El peso específico de cada uno de estos tres criterios será del 20\% para la elección del tema, otro 20\% para la planificación, y un 60\% para el desarrollo del trabajo.

6.- EnTREgA DEL TFG

El TFG debe ser defendido y evaluado una vez que se tenga constancia fehaciente de que la o el estudiante ha superado todas las materias restantes del plan 
de estudios y dispone, por tanto, de todos los créditos necesarios para la obtención del título de Grado, salvo los correspondientes al propio TFG. En cualquier caso es requisito indispensable estar matriculado/a en la asignatura "Trabajo Fin de Grado".

El TFG se entregará en la secretaría del centro en las fechas que en cada caso se indicarán, que serán alrededor de la primera semana del mes en que se desee defender, si bien cabrá completarlo hasta cinco días naturales antes de la fecha de la defensa. Además del trabajo impreso se deberá entregar en formato informático PDF una copia del mismo. Asimismo se adjuntará un documento de evaluación del proceso de elaboración firmado por el director/a del trabajo (Anexo 3).

\section{7.- TRIBUnal De EVAluación}

Cada TFG es evaluado por un tribunal de evaluación constituido al efecto, formado por tres miembros del profesorado adscrito al centro, que ocuparán la presidencia, secretaría y vocalía. En caso de ausencia de alguno de ellos le sustituirá el miembro suplente.

La secretaría la ejerce el director/a del TFG; las/os otros miembros serán especialistas en el campo de estudio de que se trate y serán designados por el profesor/a coordinadora del módulo del TFG teniendo en cuenta sus obligaciones docentes, investigadoras y de gestión. Se procurará que un mismo profesor/a no figure reiteradamente en estas comisiones.

\section{8- EXPOSICIÓN Y DEFENSA DEL TFG}

Una vez designados los miembros del tribunal de evaluación del TFG, el alumno/a será convocado para la exposición y defensa del mismo.

La exposición, que se desarrollará en sesión pública, consistirá en la explicación oral del TFG durante un período máximo de 20 minutos.

En la exposición oral se valorará especialmente que:

- El mensaje a transmitir es ordenado, de forma que existe inicio, desarrollo y final.

- La exposición ha sido clara.

- El tono de voz, los gestos y movimientos corporales son adecuados al mensaje.

- La información suministrada ha sido correcta y pertinente.

- La argumentación o razonamiento seguido es convincente.

- Se utiliza terminología propia y precisa. 
- Se han utilizado de forma adecuada recursos audiovisuales (transparencias, powerpoint, etc.).

- Se ha respondido adecuadamente a las preguntas formuladas por los miembros de la comisión evaluadora.

\section{9.- Criterios de evaluación del TFG}

Una vez finalizada la exposición, el tribunal de evaluación debatirá sobre el TFG con el autor/a durante un tiempo máximo de 20 minutos, formulando para ello tantas preguntas y aclaraciones como estime oportunas para juzgar la calidad del mismo.

A continuación, el tribunal de evaluación procederá a la evaluación del TFG mediante deliberación secreta. En la calificación se valorarán de forma ponderada los siguientes aspectos:

- 30\% la calidad del documento realizado

- 30\% el desarrollo de la exposición para su defensa

El 40\% restante será la evaluación del proceso realizado en su elaboración y habrá correspondido hacerlo al director/a de dicho TFG.

A continuación se cumplimentará el acta de calificación, que será realizada y publicada en la forma ordinaria.

\section{Archivo y consultas Del TFG}

El TFG está protegido por la Ley de Propiedad Intelectual. En consecuencia, la titularidad de los derechos de propiedad intelectual corresponderá al alumno/a que lo haya realizado, quien en ningún caso podrá ceder tales derechos hasta que no haya realizado su defensa ante el Tribunal y obtenido la calificación definitiva del TFG.

El TFG impreso se devolverá al alumno/a, en tanto que el centro archivará el trabajo en soporte informático, quedando disponible para su consulta.

\section{ANÁLISIS Y MEJORA}

Finalizado el curso académico el profesor/a coordinador del módulo del TFG presentará ante la comisión de ordenación académica del centro un informe que incluya los aspectos más relevantes en el desarrollo de los TFG. En concreto:

- Número de TFG inscritos.

- Número de TFG presentados.

- Relación de alumnado implicado. 
- Relación de profesorado en la dirección de TFG.

- Relación de profesorado ejerciendo en las comisiones de evaluación.

- Resumen de calificaciones.

- Incidencias ocurridas.

- Observaciones.

- Aspectos a mejorar.

Consecuencia de este informe, la comisión de ordenación académica podrá proponer modificaciones en la normativa de TFG ante la junta de centro. Tanto el informe como las propuestas de la comisión serán públicos.

\section{2.- Bibliografía}

Adamson, A (1990) A Student's Guide for Assignments, Projects and Research, Oxford Allison, B \& Race, P (2004) The Student's Guide to Preparing Dissertations and Theses, London, Routledge

Bell, J. (2005), Doing your Research Project: a Guide for First-Time Researchers in Education, Health and Social Science, 4th edn, Buchingham, Open University Press

Burnett, J (2009) Doing Your Social Science Dissertation, London, Sage

Bryman, A (2008) Social Research Methods, Oxford: Oxford University Press - comes with a companion web site

Gilbert, N (ed) (2005) Researching Social Life, London, Sage

Glatthorn, A \& Joyner, R (2005) Writing the Winning Thesis or Dissertation, London, Sage

Gray, D E (2004) Doing Research in the Real World, London, Sage

Hammersley, M (ed) (1993) Social Research, London, Sage

Hunt, A (2005) Your Research Project: How to Manage It, London, Routledge May, T (2001) Social Research: Issues, Methods and process, Buckingham, Open University Press

Thamesman Berry, R (2004) The Research Project: How to Write It, London, Routledge

Walliman, N (2001) Your Research Project: A step-by-step Guide for the First-time Researcher, London, Sage

Wyse, D (2006) The Good Writing Guide for Education Students, London, Sage 
ANEXO 1. OFERTA DE TRABAJOS FIN DE GRADO (DEPARTAMENTOS).

ANEXO 2. PROPUESTA DE TRABAJO FIN DE GRADO.

ANEXO 3. EVALUACIÓN DEL PROCESO DE ELABORACIÓN DEL TRABAJO FIN DE GRADO.

ANEXO 4. INFORME DE ACTIVIDAD. 


\section{ANEXO 1. OFERTA DE TRABAJOS FIN DE GRADO (DEPARTAMENTOS)}

(a remitir por el/la representante del departamento en el centro)

\section{NOMBRE DEL DEPARTAMENTO}

TÍTULO DEL TRABAJO

(a rellenar por el profesor/a que será director/a del TFG)

Resumen del Trabajo

(a rellenar por el profesor/a que será director/a del TFG) 
ANEXO 2. GRADUA BUKATZEKO LANAREN PROPOSAMENA / PROPUESTA DE TRABAJO FIN DE GRADO

\section{IKASLEAREN DATUAK / DATOS DEL/LA ALUMNO/A}

IZEN eta ABIZENAK/NOMBRE Y APELLIDOS:

NAN/DNI:

E-MAIL (UPV/EHU):

TÍTULO DEL TRABAJO

(Ikasleak betetzeko/a rellenar por el alumno/a)

\section{LANAREN LABURPENA/RESUMEN DEL TRABAJO}

(Ikasleak betetzeko/a rellenar por el alumno/a)

Data/Fecha:

\begin{tabular}{|c|c|}
\hline $\begin{array}{c}\text { Sin./Fdo.: I kaslearena/ } \\
\text { alumno-a }\end{array}$ & Sin./F do.: Zuzendaria/Director/a \\
& Sin./F do.: Sailaren ordezkaria/R epresentante del \\
& Departamento \\
\hline
\end{tabular}


ANEXO 3. ELAVUACIÓN DEL PROCESO DE ELABORACIÓN DEL TRABAJO FIN DE GRADO

TÍTULO DEL TRABAJO :

REALIZADO POR EL ALUMNO/A :

\begin{tabular}{|c|c|}
\hline $\begin{array}{l}\text { PLANTILLA DE VALORACIÓN EN } \\
\text { REALIZACIÓN DEL TRABAJO FIN DE }\end{array}$ & $\begin{array}{l}\text { EL PROCESO DE } \\
\text { SRADO (40\% nota final) }\end{array}$ \\
\hline $\begin{array}{c}\text { 1. ELECCIÓN DEL TEMA } \\
\text { (CONCRECIÓN DEL OBJETO DE } \\
\text { ESTUDIO) }\end{array}$ & Valoración \\
\hline $\begin{array}{l}\text { - Identificación del tema: Identifica y formula claramente el } \\
\text { tema objeto de estudio a partir de la aplicación de criterios } \\
\text { claros de selección y definición operativa. }\end{array}$ & $\begin{array}{l}\text { Muy mal } 12345678910 \text { Muy } \\
\text { bien }\end{array}$ \\
\hline $\begin{array}{l}\text { - Lleva a cabo adecuadamente la búsqueda documental } \\
\text { inicial sobre el tema elegido a través de las fuentes } \\
\text { documentales más significativas. }\end{array}$ & $\begin{array}{l}\text { Muy mal } 12345678910 \text { Muy } \\
\text { bien }\end{array}$ \\
\hline $\begin{array}{l}\text { - Formula preguntas y objetivos al tutor/a que orientan } \\
\text { operativamente el trabajo. }\end{array}$ & $\begin{array}{l}\text { Muy mal } 12345678910 \text { Muy } \\
\text { bien }\end{array}$ \\
\hline 2. PLANIFICACIÓN & \\
\hline $\begin{array}{l}\text { - Ha sido capaz de planificar adecuadamente los sucesivos } \\
\text { apartados y aspectos del trabajo. }\end{array}$ & $\begin{array}{l}\text { Muy mal } 12345678910 \text { Muy } \\
\text { bien }\end{array}$ \\
\hline $\begin{array}{l}\text { - Ha sido capaz de explicitarlos y concretarlos } \\
\text { adecuadamente. }\end{array}$ & $\begin{array}{l}\text { Muy mal } 12345678910 \text { Muy } \\
\text { bien }\end{array}$ \\
\hline $\begin{array}{l}\text { - Ha secuenciado temporalmente de forma adecuada las } \\
\text { diferentes partes del trabajo. }\end{array}$ & $\begin{array}{l}\text { Muy mal } 12345678910 \text { Muy } \\
\text { bien }\end{array}$ \\
\hline $\begin{array}{l}\text { - Ha presentado el trabajo justificando de forma adecuada } \\
\text { su contendido y de forma coherente con el proceso } \\
\text { general y con el proyecto inicial. }\end{array}$ & $\begin{array}{l}\text { Muy mal } 12345678910 \text { Muy } \\
\text { bien }\end{array}$ \\
\hline 3. DESARROLLO & \\
\hline $\begin{array}{c}\text { - Ha seleccionado adecuadamente las fuentes documentales } \\
\text { para la elaboración del marco teórico o la justificación del } \\
\text { tema del trabajo y ha relacionado la documentación con } \\
\text { los objetivos. }\end{array}$ & $\begin{array}{l}\text { Muy mal } 12345678910 \text { Muy } \\
\text { bien }\end{array}$ \\
\hline $\begin{array}{l}\text { - Ha integrado adecuadamente todos los conceptos } \\
\text { manejados en la elaboración del marco teórico o } \\
\text { justificación del trabajo. }\end{array}$ & $\begin{array}{l}\text { Muy mal } 12345678910 \text { Muy } \\
\text { bien }\end{array}$ \\
\hline $\begin{array}{l}\text { - Ha recogido, analizado e interpretado adecuadamente } \\
\text { los datos empíricos, prácticos o bibliográficos objeto del } \\
\text { trabajo. }\end{array}$ & $\begin{array}{l}\text { Muy mal } 12345678910 \text { Muy } \\
\text { bien }\end{array}$ \\
\hline
\end{tabular}




\begin{tabular}{|c|l|}
\hline $\begin{array}{c}\text { - Ha relacionado correctamente los datos teóricos } \\
\text { y empíricos/prácticos en función de los objetivos } \\
\text { planteados. }\end{array}$ & $\begin{array}{l}\text { Muy mal } 12345678910 \text { Muy } \\
\text { bien }\end{array}$ \\
\hline $\begin{array}{c}\text { - Ha elaborado conclusiones de manera sintética y } \\
\text { constructiva, destacando las limitaciones del estudio, } \\
\text { planteando posibles investigaciones futuras y proponiendo } \\
\text { una aplicación práctica de los resultados. }\end{array}$ & $\begin{array}{l}\text { Muy mal } 12345678910 \text { Muy } \\
\text { bien }\end{array}$ \\
\hline $\begin{array}{c}\text { Ha incorporado adecuadamente al trabajo los cambios } \\
\text { planteados en las sesiones de tutorización. }\end{array}$ & $\begin{array}{l}\text { Muy mal } 12345678910 \text { Muy } \\
\text { bien }\end{array}$ \\
\hline $\begin{array}{c}\text { En el proceso de redacción de la memoria, así como en el } \\
\text { transcurso de la interacción comunicativa con el tutor/a, } \\
\text { manifiesta un uso verbal adecuado de la terminología } \\
\text { científica propia del tema de estudio y el área de las } \\
\text { relaciones laborales en el que se inscribe. }\end{array}$ & $\begin{array}{l}\text { Muy mal } 12345678910 \text { Muy } \\
\text { bien }\end{array}$ \\
\hline
\end{tabular}

NOTA DEL PROCESO DE ELABORACIÓN DEL TFG`=

En Leioa, a

Fdo.: Nombre y apellidos del director/a del TFG.

\section{Observaciones}

9. La nota del proceso de elaboración del TFG será el resultado de (N.M. elección del tema) (.20) + (N.M. Planificación) (.20) + (N.M. Desarrollo) (.60)., y equivale al 40\% de la nota final. 
ANEXO 4. INFORME DE ACTIVIDAD

TRABAJO FIN DE GRADO

Alumno/a:

Presidencia:

Secretaría:

Vocalía:

Incidencias ${ }^{(1)}$

Observaciones / Aspectos a mejorar

Fdo.: Secretario/a del Tribunal de Evaluación 